\title{
Trabajadores con discapacidad: problemas, retos y principios de actuación en salud ocupacional
}

\author{
Workers with disabilities: Concerns, challenges, and intervention principles in \\ occupational health
} \author{
Carlos. Madrid. España. \\ Correspondencia: \\ Carlos María Alcover de la Hera \\ Paseo de los Artilleros, s/n. \\ 28032 Madrid. España. \\ Tfno.: +34 914487754 \\ E-mail: carlosmaria.alcover@uric.es
}

Carlos María Alcover de la Hera, Vanesa Pérez Torres

Unidad de Psicología Social. Departamento de Psicología. Facultad de Ciencias de la Salud, Universidad Rey Juan

Resumen

La participación y normalización de las personas con discapacidad en el mercado de trabajo depende tanto de factores externos a la organización, como el contexto legal, económico u ocupacional, como de factores relacionados con la gestión de las personas en las empresas, entre los que destacan la cultura organizacional, las políticas y prácticas de recursos humanos, los estilos de supervisión y dirección y las relaciones entre sus miembros. Este artículo tiene como objetivo revisar los principales factores psicosociales relacionados con el acceso, la integración y el desempeño de las personas con discapacidad en las organizaciones y sus implicaciones para el bienestar, la salud ocupacional y la calidad de vida laboral de este colectivo. A partir del modelo teórico propuesto por Stone y Colella ${ }^{1}$ analizamos los resultados de las principales investigaciones sobre los factores macroorganizacionales e interpersonales y su relación con los riesgos psicosociales y su prevención. Finalmente, se ofrecen una serie de conclusiones y de propuestas de intervención e investigación futura dirigidas a la mejora de la salud ocupacional de los trabajadores con discapacidad.

Palabras clave: trabajadores con discapacidad, diversidad, factores psicosociales, salud ocupacional.

\begin{abstract}

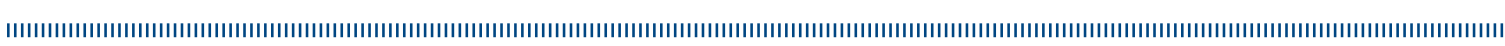

Participation and normalization of people with disabilities in the labor market are related to factors external to the organization as the legal, financial or occupational contexts, as well as factors related to people management in companies: organizational culture, HRM policies and practices, supervision and leadership styles and co-workers relationships. This paper aims to review the main psychosocial factors related to access, integration and performance of people with disabilities in organizations and their implications for wellbeing, occupational health and quality of work life of this colective. Based on the theoretical model proposed by Stone and Colella ${ }^{1}$ we analyze the research findings about macro-organizational and interpersonal factors and their relations to psychosocial risks and their prevention. Finally, we offer a set of conclusions and proposals for intervention and future research aimed at improving the occupational health of workers with disabilities.
\end{abstract}

Key words: workers with disabilities, diversity, psychosocial factors, occupational bealth. 


\section{INTRODUCCIÓN}

La discapacidad en los lugares de trabajo se ha considerado tradicionalmente de manera negativa o problemática, utilizándose enfoques basados en conceptos como estereotipo, prejuicio, hándicap, estigma, incapacidad o discriminación ${ }^{2,3}$, 4. Como resultado de ello las políticas y prácticas organizacionales y las disposiciones legales y normativas se han orientado hacia la compensación, la protección, la adaptación o los riesgos específicos asociados con este colectivo de la fuerza de trabajo. Sin embargo, en los últimos años y vinculado al movimiento más amplio dirigido a valorar la diversidad en las organizaciones, han surgido modelos y teorías que tratan la discapacidad de modo más positivo 5, 6, 4. Así, desde una perspectiva integradora o sistémica se analiza la variabilidad -evitando valoraciones positivas o negativas- en cómo las personas con diferentes discapacidades interactúan y afrontan las demandas y situaciones de trabajo en función de los recursos, el apoyo y las respuestas que brindan tanto la organización como los compañeros y el grupo de trabajo ${ }^{7,8,1,9}$.

La participación de las personas con discapacidad en la fuerza de trabajo de un país concreto depende de un gran número de factores. Como todo grupo minoritario que puede ser incluido en la denominada gestión de la diversidad, los condicionantes no solo se refieren a los habituales dentro de las organizaciones -factores individuales, grupales y de gestión/dirección-, sino también a los que Shore y sus colaboradores ${ }^{4}$ consideran dependientes de los contextos externos a las organizaciones, entre los que se encuentran la cultura nacional, el contexto ocupacional, el industrial, el legal, el familiar/comunitario y el económico; y los relacionados con dos tipos de resultados: los resultados del trabajo o productivos, en los que se incluyen los resultados organizacionales, los del grupo de trabajo y los individuales, y los resultados ajenos al trabajo, como son los familiares/ comunitarios y los sociales. Todos ellos interactúan entre sí, creando dinámicas complejas que influyen en los diferentes niveles según ciclos de retroalimentación positiva o negativa.

Esta multicausalidad dificulta que las medidas o acciones concretas tengan un efecto directo o inmediato, ya que ningún factor por sí solo suele provocar los cambios deseados con independencia del resto de agentes que confluyen en los contextos de trabajo. Un ejemplo de ello puede encontrarse en el limitado (aunque, sin duda, importante) efecto que las medidas legales dirigidas a la protección y evitación de la discriminación de las personas con discapacidad en su acceso al empleo tienen en la práctica ya que interactúan con las percepciones, atribuciones, actitudes, evaluaciones y decisiones que llevan a cabo los responsables de recursos humanos en las organizaciones, desde los procesos de selección a los de evaluación del rendimiento, formación o promoción ${ }^{10-14}$. Y no solo esto, sino que también las percepciones, actitudes y valoraciones de los compañeros o del grupo de trabajo pueden influir negativamente sobre las expectativas, la capacidad percibida y el rendimiento de las personas con discapacidad, todo lo cual a su vez puede provocar efectos reales sobre las autopercepciones y los juicios acerca de sí mismas, afectando negativamente a los niveles de autoeficacia y de ajuste emocional y al rendimiento de los trabajadores con discapacidad ${ }^{13,15}$.

Las personas con discapacidad suelen encontrarse subempleadas o desempleadas en comparación con las personas sin discapacidad, y las tasas de ocupación en la mayoría de los países continúan siendo desproporcionadas respecto a la población general ${ }^{11}$. Incluso algunos datos señalan que los niveles de empleo de las personas con discapacidad están disminuyendo en los últimos años ${ }^{15}$. En el caso de las discapacidades de origen psiquiátrico las tasas de desempleo suelen alcanzar el $90 \%{ }^{16}$, y también son muy elevadas cuando se trata de personas que presentan discapacidades severas ${ }^{17}$, lo que aún se agrava más cuando éstas también son de naturaleza mental ${ }^{18}$.

Por otro lado, la proporción de trabajadores con discapacidad declarada varía enormemente de unas ocupaciones a otras, encontrándose infrarrepresentados en ciertas ocupaciones y sobrerrepresentados en otras ${ }^{19}$. Datos procedentes de la población 
trabajadora norteamericana muestran que, incluso teniendo en cuenta su menor nivel medio -en términos generales- de formación y cualificación, las personas con discapacidad se encuentran relegadas de una manera desproporcionada a un acceso al mercado de trabajo a través de ocupaciones en las que no se valoran las competencias mejor remuneradas, como son las relacionadas con habilidades de información, conocimiento y comunicación, además de tratarse de empleos en los que no se requiere habitualmente poseer experiencia previa. A su vez, el frecuente subempleo al que se ven obligadas las personas con discapacidad conlleva para ellas la percepción de bajos salarios y una menor seguridad y estabilidad laboral ${ }^{19}$.

En España, a partir del Real Decreto 1971/1999, de 23 de diciembre, por el que se regula el procedimiento para el reconocimiento, declaración y calificación de la minusvalía, se entiende por persona con discapacidad aquella cuyas posibilidades de integración educativa, laboral o social se encuentran disminuidas como consecuencia de una deficiencia, previsiblemente permanente, de carácter congénito o no, en sus capacidades físicas, psíquicas o sensoriales. A efectos de la inclusión en las medidas protectoras derivadas de la condición de persona con discapacidad se consideran en tal situación las personas en edad laboral (16 años) afectadas, como mínimo, por una disminución en su capacidad física o psíquica del 33\%, con las dificultades consiguientes para el acceso al mercado laboral, el mantenimiento de un empleo y el desarrollo de una carrera profesional plena.

Según los datos del Comité Español de Representantes de Personas con Discapacidad (CERMI), en España hay más de cuatro millones de personas con algún tipo de discapacidad, que junto a las que constituyen su entorno familiar suponen alrededor de doce millones de ciudadanos ${ }^{20}$. Los datos más actuales disponibles sobre las tasas de empleo de las personas con discapacidad proceden de la Encuesta sobre Discapacidad, Autonomía Personal y situaciones de Dependencia (EDAD 2008), realizada entre el mes de noviembre de 2007 y febrero de 2008 en domicilios familiares. Como se señala en el Informe Olivenza 2010, elaborado por el Observatorio Español de la Discapacidad ${ }^{21}$, la realidad reflejada en los datos de esta Encuesta tiene que matizarse no solo por los años transcurridos, sino también por el agravamiento de la crisis económica y financiera sufrida en España desde entonces, que ha incrementado significativamente los niveles generales de desempleo, afectando con mayor incidencia a los colectivos más desfavorecidos, entre ellos las personas con discapacidad. La Tabla 1 resume los principales datos al respecto.

Tabla I. Tasas de actividad, empleo y paro en personas con y sin discapacidad y población total en edad laboral (16- 64 años), por sexo. España 2008

\begin{tabular}{|c|c|c|c|c|}
\hline & & $\begin{array}{l}\text { Personas con } \\
\text { discapacidad }\end{array}$ & $\begin{array}{l}\text { Personas sin } \\
\text { discapacidad }\end{array}$ & $\begin{array}{c}\text { Población } \\
\text { total }\end{array}$ \\
\hline \multirow{3}{*}{ 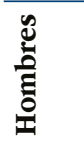 } & Tasa de actividad & $40,3 \%$ & $84,7 \%$ & $82,7 \%$ \\
\hline & Tasa de empleo & $33,4 \%$ & $77,4 \%$ & $75,4 \%$ \\
\hline & Tasa de paro & $17,2 \%$ & $8,6 \%$ & $8,8 \%$ \\
\hline \multirow{3}{*}{ 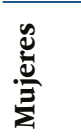 } & Tasa de actividad & $31,2 \%$ & $65,3 \%$ & $63,6 \%$ \\
\hline & Tasa de empleo & $23,7 \%$ & $56,6 \%$ & $54,9 \%$ \\
\hline & Tasa de paro & $24,0 \%$ & $13,4 \%$ & $13,6 \%$ \\
\hline \multirow{3}{*}{ 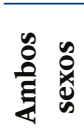 } & Tasa de actividad & $35,5 \%$ & $75,2 \%$ & $73,2 \%$ \\
\hline & Tasa de empleo & $28,3 \%$ & $67,2 \%$ & $65,3 \%$ \\
\hline & Tasa de paro & $20,3 \%$ & $10,6 \%$ & $10,9 \%$ \\
\hline
\end{tabular}

Fuente: Informe Olivenza 2010, a partir de los datos del INE, Encuesta de Discapacidad, Autonomía personal y situaciones de Dependencia, EDAD 2008 (Módulo de hogares).

Por lo que respecta a los tipos de contratos que se realizan a las personas con discapacidad, el Informe Olivenza 2010 ofrece los siguientes datos, recogidos en la Tabla 2. 
Tabla II. Contratos realizados a personas con discapacidad, según tipo de contrato. 2008, 2009 y 2010 (enero-agosto)

\begin{tabular}{lrrrrrrr}
\hline & \multicolumn{2}{c}{ AÑO 2008} & \multicolumn{2}{c}{ AÑO 2009 } & \multicolumn{2}{c}{ AÑo 2010 } \\
\multicolumn{1}{c}{ TIPOS DE CONTRATOS } & Acumulado anual & \multicolumn{2}{c}{ Acumulado anual } & \multicolumn{2}{c}{ Acumulado a 01/09 } \\
& \multicolumn{1}{c}{$\mathbf{N}$} & \multicolumn{1}{c}{$\%$} & \multicolumn{1}{c}{ N } & \multicolumn{1}{c}{$\%$} & \multicolumn{1}{c}{ N } & \multicolumn{1}{c}{$\%$} \\
\hline Indefinido ordinario (Bonif./No bonif.) & 728 & $1,31 \%$ & 751 & $1,46 \%$ & 465 & $1,18 \%$ \\
Indefinido F.C.I* & 3.082 & $5,53 \%$ & 2.323 & $4,50 \%$ & 1.467 & $3,72 \%$ \\
Indefinido personas con discapacidad & 10.431 & $18,72 \%$ & 7.966 & $15,44 \%$ & 5.370 & $13,60 \%$ \\
Obra o servicio & 7.665 & $13,76 \%$ & 7.316 & $14,18 \%$ & 6.374 & $16,15 \%$ \\
Eventual & 7.436 & $13,35 \%$ & 8.445 & $16,37 \%$ & 6.897 & $17,47 \%$ \\
Interinidad & 6.074 & $10,90 \%$ & 7.334 & $14,22 \%$ & 5.861 & $14,85 \%$ \\
Temporal personas con discapacidad & 15.327 & $27,51 \%$ & 13.135 & $25,47 \%$ & 9.539 & $24,17 \%$ \\
Prácticas & 7 & $0,01 \%$ & 6 & $0,01 \%$ & 14 & $0,04 \%$ \\
Formación & 563 & $1,01 \%$ & 620 & $1,20 \%$ & 313 & $0,79 \%$ \\
Otros contratos & 98 & $0,18 \%$ & 132 & $0,26 \%$ & 88 & $0,22 \%$ \\
Convertidos en indefinidos & 4.303 & $7,72 \%$ & 3.549 & $6,88 \%$ & 2.939 & $7,45 \%$ \\
\hline TOTAL & 55.714 & $100,00 \%$ & 51.577 & $100,00 \%$ & 39.471 & $100,00 \%$ \\
\hline Indefinidos & 18.544 & $33,28 \%$ & 14.589 & $28,28 \%$ & 10.241 & $25,95 \%$ \\
Temporales y otros & 37.170 & $66,72 \%$ & 36.988 & $71,72 \%$ & 29.230 & $74,05 \%$ \\
\hline
\end{tabular}

Fuente: Informe Olivenza 2010, a partir de los datos del Servicio Público de Empleo Estatal.

En opinión del OED, el mantenimiento del dinamismo en la contratación de personas con discapacidad, pese a la situación de crisis, indica que las medidas incluidas en el Plan extraordinario de mantenimiento y fomento del empleo de los trabajadores con discapacidad establecido por la Ley $27 / 2009$, de 30 de diciembre, de medidas urgentes para el mantenimiento y el fomento del empleo y la protección de las personas desempleadas, están teniendo un efecto positivo. No obstante, es importante señalar que estos datos sólo muestran el volumen de las contrataciones efectuadas, y no permiten obtener conclusiones acerca de la evolución de variables como las tasas de actividad, empleo y desempleo, que son esenciales para el conocimiento de la verdadera situación de empleo de las personas con discapacidad. En consecuencia, el Informe Olivenza 2010 considera necesario que la variable discapacidad se incorpore en las estadísticas de empleo, y en particular en la Encuesta de Población Activa y en las series estadísticas sobre afiliados en situación de alta laboral que difunde la Seguridad Social ${ }^{21}$.

De forma muy similar a lo que ocurre en otros países y $\operatorname{contextos}^{22,23,13,25}$, los problemas de acceso y de inserción en el mercado laboral de las personas con discapacidad en España responden a una diversidad de factores, que, según el CERMI ${ }^{25}$, pueden resumirse en los siguientes:

- Un nivel educativo y una cualificación profesional deficiente o no adaptada a las demandas y necesidades reales del sistema productivo.

- Falta de motivación e información de la propia persona con discapacidad y de sus entornos familiares, que se traduce en dificultades para mejorar su cualificación profesional o desarrollar habilidades de búsqueda de empleo.

- Actitud inicial negativa de una parte de los empleadores o de los responsables de recursos humanos, que revela un prejuicio inicial hacia las capacidades potenciales de la persona con discapacidad o una serie de atribuciones, estereotipos e ideas falsas preconcebidas (baja capacidad de aprendizaje, escasa polivalencia, difícil reciclaje, altos costes por la adaptación de lugares y puestos de trabajo, etc.).

- Dificultades para acceder a fuentes de financiación de cara a crear su propio negocio, lo que limita su capacidad emprendedora.

- Dificultades de accesibilidad (transporte, adaptación de centros de formación o de trabajo) que pueden obstaculizar o encarecer la integración laboral. 
En definitiva, la integración plena y la normalización -es decir, la similaridad de las condiciones de trabajo con los trabajadores sin discapacidad en todos los términos posibles-, de las personas con discapacidad en el mercado laboral se enfrenta a una serie de dificultades y de desafíos ${ }^{26}$, que se suman a los de la población general en situaciones de crisis como la actual. Al mismo tiempo, la conciencia, la sensibilidad y la reivindicación de esta necesidad de normalización se incrementa tanto en el colectivo de personas con discapacidad como en las familias, las comunidades, los agentes sociales y las sociedades en su conjunto, lo que ayuda a contrapesar los problemas señalados con oportunidades, propuestas y acciones desarrollados por los legisladores y los poderes públicos, así como por los profesionales que trabajan desde disciplinas como la Psicología de la Salud Ocupacional.

El objetivo de este artículo es revisar los principales factores psicosociales que intervienen en la integración y en el desenvolvimiento de los trabajadores con discapacidad en contextos organizacionales normalizados, o de integración ordinaria, sin incluir los contextos laborales orientados a la integración especial ${ }^{27}$, también denominado empleo protegido. A partir del modelo general formulado por Dianna Stone y Adrienne Colella ${ }^{1}$, diferenciamos dos niveles de factores: macroorganizacionales e interpersonales, los cuales interactúan con los factores individuales y las características específicas de cada tipo de discapacidad, y analizamos los elementos más relevantes de cada uno de ellos. Adicionalmente, identificamos los principales riesgos de carácter psicosocial con mayor incidencia en este colectivo, así como algunas medidas para su prevención. Por último, se ofrecen una serie de conclusiones y de propuestas de intervención dirigidas a la mejora de la salud ocupacional de los trabajadores con discapacidad.

\section{FACTORES MACROORGANIZACIONALES}

Dentro de las condiciones que pueden conducir a la presencia de riesgos psicosociales en los trabajadores se encuentran los denominados factores macroorganizacionales. En este apartado analizamos específicamente los resultados de las investigaciones relacionadas con la cultura organizacional, el estilo de dirección y el liderazgo, así como las políticas y prácticas de la empresa que afectan a los trabajadores con discapacidad.

\section{Cultura organizacional, estilo de dirección y liderazgo}

Como señalamos más arriba, uno de los principales modelos teóricos sobre los factores organizacionales que afectan a los trabajadores con discapacidad fue propuesto por Stone y Colella ${ }^{1}$, reflejado en la Figura 1. De acuerdo con esta propuesta teórica factores como la legislación, las características de la organización, de los compañeros y supervisores, los atributos de los trabajadores y la naturaleza del trabajo tienen influencia sobre el trato recibido, las oportunidades de formación y desarrollo, la inclusión en grupos de trabajo y la manifestación de conductas de ayuda hacia las personas con discapacidad.

Las variables consideradas en este modelo con mayor impacto en las personas con discapacidad incluyen las normas y valores organizacionales, las políticas de recursos humanos y la naturaleza del sistema de recompensas. Stone y Colella ${ }^{1}$ predicen en su modelo que aquellas organizaciones más flexibles y que apoyan a sus empleados favorecen la incorporación y adaptación de las personas con discapacidad a su puesto de trabajo. Por otro lado, en aquellas organizaciones donde predominan valores de igualdad y estandarización en los puestos de trabajo (más burocráticas), los directivos pueden encontrarse ante dilemas o situaciones contradictorias, ya que no podrían brindar un trato igualitario a los trabajadores con discapacidad. Al mismo tiempo, al necesitar éstos una adaptación en mayor o menor medida de sus puestos de trabajo, esta acción podría ser interpretada por el resto de empleados como un acto injusto o poco equitativo -es decir, como un acto de discriminación positiva- o percibida como un desequilibrio de la justicia procedimental ${ }^{28}$. Además, valores sociales como el desempeño competitivo o el 
individualismo pueden afectar negativamente en cómo se perciben las habilidades de las personas con discapacidad. Por el contrario, las organizaciones donde predominan valores de justicia social, igualdad, cooperación y ayuda, tienden a favorecer la percepción acerca de las contribuciones que pueden aportar los trabajadores con discapacidad a la organización. No obstante, los datos no son concluyentes, puesto que parece que las culturas individualistas son más proclives a llevar a cabo políticas activas para incrementar la presencia de personas con discapacidad en el mundo laboral que las culturas colectivistas $^{29}$, lo que podría indicar la influencia de aspectos culturales más amplios que los valores organizacionales.

Figura 1. Modelo de los factores que afectan el trato de los individuos con discapacidad dentro de las organizaciones

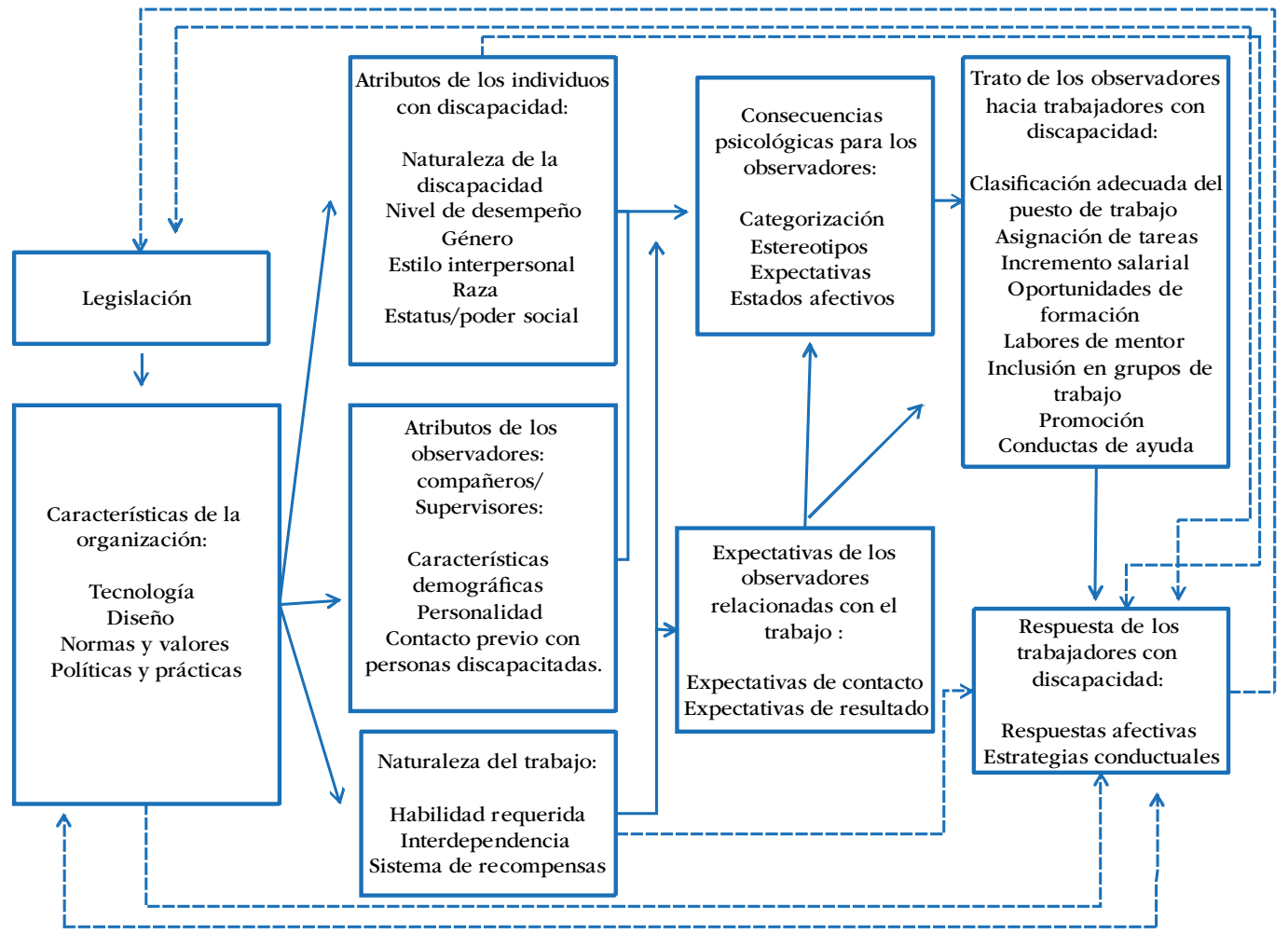

Fuente: Stone y Colella (1), p. 355.

Estas variables incluidas en el modelo de Stone y Colella ${ }^{1}$ forman parte de la cultura organizacional (valores, normas y formas de comportamiento compartidos por los miembros de la organización) cuya importancia radica en su influencia sobre la transmisión de valores compartidos, la diferencia o igualdad entre los empleados así como en la prescripción de comportamientos apropiados en la interacción social y en las políticas y prácticas que se adoptan en relación con la diversidad ${ }^{30}$. En un estudio de carácter cualitativo, Gilbride, Stensrud, Vandergoot y Golden ${ }^{31}$ identificaron los factores específicos que caracterizan a las empresas abiertas a la inclusión de personas con discapacidad, entre los que destacan: la igualdad de trato, la cultura inclusiva, un estilo directivo personal y flexible, énfasis en el desempeño del trabajador y no en su discapacidad, así como la disposición de proveer las adaptaciones necesarias para todos los empleados.

Schur, Kruse y Blanck ${ }^{32}$ realizaron un análisis de la influencia que tiene la cultura corporativa en el acceso al empleo, a las oportunidades de desarrollo profesional y al diseño de un entorno de apoyo que beneficie no solo las personas con discapacidad, sino también al resto de empleados de la organización. Estos investigadores señalan que la cultura corporativa puede crear barreras actitudinales, conductuales y físicas, o por el contrario, facilitar el desempeño laboral de este colectivo. Por ejemplo, el compromiso de incorporar personal con discapacidad puede ser parte de los valores compartidos, aunque quizá no sea un patrón de comportamiento interiorizado por todos los miembros de la 
organización. Esto se puede reflejar en el nivel más visible de la empresa al no favorecer las adaptaciones necesarias para el acceso a personal discapacitado, el tipo de empleo ofrecido o si los supervisores y compañeros manifiestan actitudes negativas. Además, destacan la importancia que tienen los valores y las prácticas organizacionales en el trato que reciben las personas con discapacidad. Coinciden con Stone y Colella ${ }^{1}$ en la importancia de favorecer un ambiente de apoyo y de respeto para la diversidad. De acuerdo con Triana, García y Colella ${ }^{33}$ el apoyo organizacional a la diversidad es la percepción que los empleados tienen sobre las acciones que realiza la empresa para promover los valores de diversidad. Este apoyo no solo incluye la identidad personal y grupal de los miembros de la organización, sino también las actitudes individuales y las acciones de los directivos hacia los grupos minoritarios ${ }^{33}$.

Estos valores deben reflejarse en las políticas y prácticas de la organización para integrar a las personas con discapacidad ${ }^{32,30}$. Como señala Spataro ${ }^{30}$ es importante que la alta dirección esté atenta al sistema de valores subyacente a la cultura y que sea transmisora de la contribución que realizan los trabajadores con discapacidad.

De esta forma, tanto el estilo de dirección como el estilo de liderazgo influyen sobre la manera de abordar la inclusión y el trato de las personas con discapacidad en la empresa. Cubero ${ }^{34}$ sugiere que un estilo de liderazgo cercano, como el situacional, puede impulsar la igualdad en el lugar de trabajo y mejorar la percepción de los trabajadores con discapacidad.

“(...) las personas con discapacidad pueden sentirse cómodas con un líder centrado en las relaciones..., con buena disposición hacia los trabajadores..., que contemple las necesidades individuales... y que provea del apoyo necesario dependiendo de la situación. Esta flexibilidad del líder que utiliza un estilo situacional tiene importantes implicaciones en aquellos trabajadores con discapacidad que necesitan autonomía en su puesto u orientación específica dependiendo de su nivel de disposición a cumplir con las tareas asignadas" ${ }^{34}$.

Por lo tanto, fomentar una cultura inclusiva y orientada a la diversidad, donde predomine un estilo de dirección participativo debería ser uno de los primeros pasos en la prevención de riesgos psicosociales en trabajadores con discapacidad.

\section{Políicas y prácticas de la empresa}

Las políticas y prácticas de la empresa, especialmente las relacionadas con los procedimientos de reclutamiento y selección y la evaluación del rendimiento, están influidas por la cultura organizacional y tienen un efecto directo sobre el trato que reciben los trabajadores con discapacidad ${ }^{22,1}$.

Los estudios realizados en el ámbito de la selección y contratación de personas con discapacidad exploran la influencia de la naturaleza o tipo de discapacidad de los candidatos a los puestos ${ }^{22,13}$ y las expectativas del futuro empleador sobre los trabajadores con discapacidad ${ }^{23}$ 24. Algunas investigaciones sugieren que existe una jerarquía de preferencias hacia diferentes tipologías de discapacidad en los procesos de contratación, por ejemplo, aquellas personas con discapacidad física son valoradas de forma más favorable que los que poseen discapacidad cognitiva -disfunciones mentales o de aprendizaje-, o emocional -depresión u otros trastornos- ${ }^{22,35,36,6,1}$. Se han dado algunas explicaciones al respecto, como la elección de un tipo de discapacidad más visible o la influencia de los estigmas asociados a algunas tipologías, especialmente las de tipo psiquiátrico $^{22,36}$. Otros estudios ${ }^{37,32}$ han mostrado que la experiencia o el contacto con personas con discapacidad por parte de los encargados de los procesos de selección influye durante la realización de las entrevistas y en el proceso posterior de decisión, indicando que la ausencia de contacto previo sesga negativamente las percepciones y los juicios.

En el contexto español, un estudio realizado en los años de 2006 y 2007 por la Fundación ONCE y la Fundación Manpower ${ }^{39}$ sobre la situación de las personas con 
discapacidad en el entorno laboral, muestra resultados importantes en la percepción de este colectivo, la valoración de su desempeño en los puestos de trabajo, así como sobre las motivaciones y las barreras existentes para su contratación. En primer lugar, aparece una percepción diferenciada sobre la discapacidad entre los empresarios encuestados. Es decir, no existe una percepción de un colectivo único, sino que surgen diferentes categorías en función de la tipología de la discapacidad y el grado de severidad. Los resultados obtenidos a partir de los empresarios encuestados de 1.500 empresas mostraron que perciben de manera más positiva a las personas con discapacidad física, mientras que las personas con discapacidad cognitiva o emocional eran percibidas de manera más negativa. Además, existe una idea compartida por la mayoría de los participantes, según la cual las personas con discapacidad no pueden ocupar cualquier puesto de trabajo (por ejemplo, aquellos con elevada responsabilidad, intensa actividad física o de cara al público). En este sentido, se asoció a este colectivo con el desempeño de puestos de menor cualificación ${ }^{39}$, un resultado coincidente con el obtenido en otros países ${ }^{19}$.

En cuanto a las expectativas de los empleadores sobre los trabajadores con discapacidad algunas investigaciones señalan la preocupación de las empresas sobre su desempeño futuro, acerca de si podrán afrontar las demandas de los puestos de trabajo o sobre los conocimientos o habilidades que realmente poseen ${ }^{23,24}$. En este caso, una experiencia previa de éxito -en cuanto a la contratación y el rendimiento posterior- de un trabajador con discapacidad incrementa la satisfacción del empleador y aumenta la probabilidad de que vuelva a emplear personas con discapacidad en el futuro ${ }^{24}$; además influye el compromiso social expresado en la política de responsabilidad social de la empresa $^{23}$. Esto sugiere que las empresas dan mucha más importancia al desempeño individual y a los resultados del trabajador en los equipos de trabajo que a los aspectos relacionados con la discapacidad ${ }^{24}$.

Dentro de la evaluación del desempeño, algunos estudios sugieren tener en cuenta la presencia de dos tipos de juicio previo que pueden hacerse sobre los trabajadores con discapacidad. Por un lado, existe una postura favorable o positiva en la que es necesario considerar aspectos como la deseabilidad social, el paternalismo o la denominada "norma de amabilidad" según la cual existe un sesgo de valoración positiva hacia las personas con discapacidad, aún cuando sus resultados no sean adecuados. Además, los evaluadores podrían tener expectativas iniciales más bajas sobre las personas con discapacidad, por lo que la valoración se realizaría sobre estas expectativas y no sobre el desempeño real ${ }^{13,4}$. Por otro lado, también aparecen juicios negativos relacionados con la discriminación, los estereotipos y los estigmas relacionados con las personas con discapacidad. Así, cuando las personas realizan una valoración de personas con discapacidad basada en juicios negativos, suelen considerarse menos capaces y por lo tanto receptoras de expectativas de bajo desempeño ${ }^{13}$.

En relación con este último aspecto, en el estudio de la Fundación Once mencionado ${ }^{29}$ más de un $70 \%$ de los empresarios encuestados manifestaron una opinión positiva sobre el rendimiento de las personas con discapacidad. Por ejemplo, consideraron que se integraban adecuadamente a la plantilla y que su rendimiento era similar al del resto de trabajadores. Además, aquellas empresas que han tenido o tienen actualmente personas con discapacidad en su plantilla presentan una visión más favorable acerca de su desempeño en comparación con el resto de empresas encuestadas. Este resultado incide en la importancia de las experiencias reales con personas con discapacidad en el entorno laboral y su influencia en la integración de este colectivo posteriormente ${ }^{29}$. Este aspecto concuerda con uno de los factores incluido también en el modelo de Stone y Colella ${ }^{1}$, esto es, el contacto previo con personas con discapacidad. Según el modelo, aquellos supervisores o compañeros de trabajo que han tenido una experiencia anterior con personas con discapacidad (familia, amigos, compañeros de trabajo) reaccionan de manera más favorable ante este colectivo que aquellos que no la han tenido. De forma similar, Ch. Scherbaum, K. Scherbaum y Popovich ${ }^{40}$ investigaron la relación entre la calidad de la experiencia previa con compañeros con discapacidad, las expectativas relacionadas con el trabajo de esos empleados y las reacciones afectivas hacia ese 
colectivo. En sus conclusiones señalaron que las experiencias positivas con compañeros de trabajo con discapacidad se asociaban igualmente a expectativas y reacciones afectivas positivas. De forma similar, los resultados de un estudio realizado con una muestra de 189 ejecutivos pertenecientes al grupo norteamericano de firmas incluidas en Fortune $500^{41}$ mostró que las empresas con menos exposición a las personas con discapacidad tenían expectativas más negativas hacia ellas.

En definitiva, es necesario destacar cómo el diseño y la aplicación de las políticas de la empresa se traducen en prácticas favorables de inclusión que tienen en cuenta las necesidades específicas de los trabajadores con discapacidad y previenen situaciones de discriminación o de valoración sesgada sobre sus competencias y su rendimiento.

\section{FACTORES INTERPERSONALES}

\section{Actitudes, atribuciones, estereotipos y prejuicios}

Una experiencia común a las personas con discapacidad es la de enfrentarse a conductas y actitudes estigmatizadoras ${ }^{42}$. Quienes son percibidos como portadores de estigma no son responsables de él, ya que depende de aquellos que poseen el poder social, económico y político para estigmatizar a otros ${ }^{43}$. Según Link y Phelan ${ }^{43}$ el estigma tiene cuatro componentes: la distinción y el etiquetamiento de las diferencias humanas; la vinculación de las personas etiquetadas con características indeseables; la separación de "ellos" (las personas estigmatizadas) de "nosotros"; y la consiguiente pérdida de estatus y discriminación que conducen a desigualdad de oportunidades y de resultados en la vida ${ }^{43}$.

Una vez que un grupo es estigmatizado, las personas que no pertenecen a él interiorizan y asumen -habitualmente de manera acrítica- las creencias implícitas asociadas al estigma, las cuales influyen en las percepciones, atribuciones y actitudes hacia las personas estigmatizadas. Como otros factores psicosociales, este proceso de origen social se traduce en comportamientos interpersonales, de manera que las personas con discapacidad -como grupo estigmatizado- pueden enfrentarse a interacciones con compañeros de trabajo, supervisores, clientes, usuarios, gerentes o directivos en las que operan, implícita o explícitamente, estos procesos. Por ejemplo, un estudio reciente ha mostrado cómo la percepción de estigma media la relación entre la discapacidad y la aceptación de la persona que la posee, acentuando negativamente la percepción de la discapacidad cuando ésta se asocia con la pertenencia a un grupo estigmatizado ${ }^{44}$.

Es importante subrayar estos aspectos, ya que los resultados de las investigaciones muestran que algunas de las mayores dificultades para la integración y normalización de las personas con discapacidad en contextos de trabajo se encuentra en los estereotipos y las actitudes negativas de buena parte de los empleadores y de los compañeros hacia ellas y hacia su capacidad de trabajar ${ }^{45,46,47,48,13,49}$. Así, los resultados de una de las investigaciones recientes de mayor envergadura, realizada con una muestra representativa de 1.200 empresas de EE.UU. y del Reino Unido ${ }^{11}$, muestran que un $23 \%$ de los empleadores admite que las actitudes y los estereotipos negativos eran las barreras más significativas para contratar a personas con discapacidad. Este efecto negativo se manifiesta no solamente en los procesos de reclutamiento y selección, o en la adopción de medidas para la adaptación de los puestos de trabajo -ambos aspectos dependientes de la alta dirección-, sino que las actitudes, estereotipos y prejuicios hacia las personas con discapacidad suelen ser más insidiosas entre los compañeros de trabajo y los supervisores. No obstante esto último, algunos resultados de estudios realizados con empleadores ${ }^{41}$ señalan que una buena parte de ellos consideran que la incomodidad de los compañeros y los supervisores ante trabajadores con discapacidad no debería ser una barrera para su contratación, si bien no consultan acerca de ello a sus empleados antes de tomar decisiones. 
Otros estudios han encontrado diferencias en cuanto a las características y experiencias de los observadores y las actitudes hacia los trabajadores con discapacidad. Así, las mujeres y quienes han tenido experiencia previa con personas con discapacidad tienden a tener actitudes más positivas que los hombres y quienes no han tenido experiencia previa ${ }^{50,51,52}$. Un aspecto interesante es el encontrado por Popovich, Ch. Scherbaum, K. Scherbaum y Polinko relativo a las diferencias significativas entre lo que las personas consideran como discapacidad y lo que no, y que no siempre coincide con las definiciones legales ${ }^{52}$. Por ejemplo, los participantes en este estudio consideraban discapacidad el trastorno por déficit de atención, los trastornos del habla o los problemas de visión (que legalmente no lo son, al menos en EE.UU.), y no consideraban discapacidad a la diabetes, a la esquizofrenia o al alcoholismo (que legalmente sí lo son). Esto puede dar lugar a la utilización de estereotipos y al desarrollo de actitudes negativas, así como eventualmente a situaciones de discriminación, de forma inadvertida, lo que puede tener importantes consecuencias para los individuos y las organizaciones.

\section{Apoyo organizacional, apoyo del supervisor y apoyo de los compañeros}

La investigación previa muestra que las principales fuentes de apoyo social dentro del entorno laboral son los supervisores, los compañeros de trabajo $^{53}$ y la organización en sí misma a través de sus políticas y prácticas ${ }^{54}$. Este tipo de apoyo puede ser instrumental, al ayudar en la realización de las tareas, resolver dudas o facilitar las herramientas adecuadas de trabajo y también emocional, mediante la expresión de empatía, cuidado, escucha y amistad ${ }^{55}$.

En el caso de las personas con discapacidad, en la literatura especializada se utiliza el término apoyos naturales ${ }^{56}$ para referirse a aquellas personas que asumen proporcionar asistencia, información o compañía en el lugar de trabajo con el objetivo de facilitar el rendimiento independiente, total o parcialmente, de personas con discapacidad, y para quienes la prestación de esa asistencia, información o contacto no es su principal responsabilidad y es independiente de si será o no compensada por la organización ${ }^{57,58,59,56,60}$. Este enfoque ecológico ${ }^{61}$ se basa en la evidencia de que el apoyo prestado por las personas habituales o del entorno laboral es mucho más eficaz que el dispensado por personal o servicios especializados ${ }^{62}$, de manera que los programas de empleo con apoyo (supported employment) constituyen una estrategia eficaz para el logro de beneficios sociales y económicos en la integración de las personas con discapacidad en entornos normalizados ${ }^{63}$. Los resultados de la revisión realizada por Storey ${ }^{56}$ sobre los efectos de apoyos naturales como la instrucción de los compañeros (en la adquisición de habilidades sociales y de comunicación, así como en las adaptaciones y modificaciones del puesto), su apoyo en el entrenamiento en solución de problemas y en el fomento de la iniciativa, y los programas formales e informales de mentoring -al igual que los de coaching ${ }^{64}$ - muestran que estas estrategias son eficaces para potenciar los logros laborales de las personas con discapacidad en contextos de trabajo integrados, especialmente en promover el aprendizaje y mejorar el rendimiento ${ }^{61}$. De igual modo, las relaciones sociales positivas en los contextos laborales contribuyen de manera significativa a prevenir y reducir el estrés en los trabajadores con discapacidad $^{65}$.

El apoyo de la organización, de los compañeros y supervisores de trabajo han sido estudiados por su impacto positivo en el bienestar de los empleados ${ }^{54,66,55,53}$ y sobre las actitudes hacia el trabajo ${ }^{67,68}$. Es esperable que si en la población general los efectos de los distintos tipos de apoyo recibido en los contextos laborales son positivos, en el caso de los trabajadores con discapacidad sean similares o superiores.

En nuestro país, un estudio realizado por Verdugo y Jordán de Urríes ${ }^{69}$ mostró que el apoyo ofrecido por los compañeros de trabajo a un grupo de empleados con discapacidad intelectual producía una mejora en los resultados de trabajo; además, cuando eran los supervisores o gerentes los que brindaban apoyo, los beneficios obtenidos sobre la calidad de vida general, la pertenencia social, la integración en la comunidad y 
la antigüedad en el puesto de trabajo aumentaban. También los resultados obtenidos por Flores, Jenaro, Orgaz y Martín ${ }^{70}$ han mostrado que la percepción de apoyo de los supervisores y los compañeros, junto con las bajas demandas de tarea, eran buenos predictores de la calidad de vida laboral en trabajadores con discapacidad intelectual. Por su parte, los resultados de un estudio llevado a cabo por nuestro equipo recientemente ${ }^{71}$, con una muestra de 135 empleados con diferentes tipos de discapacidad que trabajaban en empresas privadas, indican que los empleados que perciben un elevado apoyo organizacional mostraban niveles elevados de satisfacción laboral; contrariamente a lo esperado, en los empleados que también percibían un elevado apoyo de los supervisores y de los compañeros la relación no fue estadísticamente significativa con la satisfacción laboral. En cuanto a las relaciones entre los tres tipos de percepción de apoyo y la intención de abandono de la organización, nuestros resultados confirmaron una relación inversa estadísticamente significativa en todos los casos, de manera que cuanto mayor era el apoyo percibido procedente de las tres fuentes, mayor era la intención de permanecer en la organización ${ }^{71}$. Estos resultados son prometedores, y suponen un primer paso para continuar profundizando en el estudio del papel que desempeña el apoyo en relación con otras conductas y actitudes de las personas con discapacidad en contextos organizacionales y laborales, y pueden contribuir de manera significativa al diseño de estrategias que faciliten y potencien la normalización de este colectivo en el mercado laboral.

\section{Apoyo familiar y apoyo social}

Aunque no se trata de un factor directamente relacionado con el contexto de trabajo, el apoyo familiar y el apoyo social dispensado por las personas de su entorno inmediato son factores importantes en la normalización de los trabajadores con discapacidad. En particular, el apoyo familiar puede ser un valioso recurso para que en la familia adquieran confianza y seguridad en sus propias competencias y para afrontar situaciones de tensión, conflicto o estrés en el trabajo. Así, el enriquecimiento trabajo-familia puede implicar tanto habilidades, destrezas o valores, de carácter más instrumental, como otros factores de naturaleza más afectiva, como el estado de ánimo, el clima o las emociones positivas ${ }^{72}$. De este modo, la percepción de apoyo familiar y del enriquecimiento trabajo-familia puede ser un protector eficaz en el afrontamiento y en las consecuencias de experiencias estresoras, actuando como un amortiguador de los potenciales efectos negativos ${ }^{73,74}$, un aspecto que sería interesante investigar específicamente en el caso de las personas con discapacidad. Sin embargo, hasta ahora la investigación sobre el apoyo familiar y social fuera del contexto organizacional en personas con discapacidad es prácticamente inexistente, y sería interesante estudiarlos en relación con las percepciones de apoyo organizacional, del supervisor y de los compañeros ${ }^{71}$ y conocer las posibles interacciones entre los distintos tipos de apoyo.

Por último, un aspecto que también reviste importancia es el referido a las posibles consecuencias negativas que puede tener la prestación de apoyo a las personas con discapacidad en entornos laborales, ya que el personal directamente implicado en este tipo de tareas suele ser más vulnerable al estrés, la depresión y al burnout ${ }^{75,76,77,}$ requiriendo a su vez medidas de apoyo por parte de la organización para realizar su trabajo adecuadamente y paliar las posibles consecuencias negativas para su bienestar y su salud ocupacional.

\section{RIESGOS PSICOSOCIALES Y SU PREVENCIÓN}

La incidencia de los riesgos de carácter psicosocial puede ser mayor en el colectivo de los trabajadores con discapacidad que en la población general ${ }^{78,79}$, y pueden afectar de manera significativa en la percepción de su satisfacción y su calidad de vida laboral ${ }^{70,80}$.

En este ámbito específico apenas se han formulado modelos ni se han realizado estudios con muestras significativas. Recientemente, Jenaro y Flores ${ }^{81}$ han propuesto un modelo que supone una primera aproximación al análisis de la calidad de vida laboral de 
las personas con discapacidad. A partir del conocido modelo de demandas-recursos en el trabajo $^{82,83}$, predicen que la percepción de desequilibrio entre las demandas laborales y los recursos disponibles genera estrés (como principal riesgo psicosocial) y supone una disminución de la calidad de vida laboral percibida, lo que tienen consecuencias tanto para el individuo -afectando a su bienestar y salud física y psicológica, su satisfacción laboral y sus relaciones sociales, dentro y fuera del contexto de trabajo- como para la organización -que se traduce en un descenso de la productividad y la calidad de los resultados de tarea y en un incremento del absentismo-. En un estudio realizado con trabajadores con discapacidad intelectual ${ }^{84}$, pertenecientes a centros especiales de empleo y programas de empleo con apoyo, los resultados mostraron que el grado de equilibrio entre las demandas procedentes de las características y la organización del trabajo, de las relaciones con compañeros y supervisores y de los factores derivados de la cultura y el clima, en interacción con los recursos disponibles, tanto personales como contextuales, para afrontarlas es un antecedente clave en la experiencia de estrés y en la consiguiente disminución de la satisfacción y la percepción de calidad de vida laboral.

En un estudio posterior ${ }^{85}$ el análisis se ha ampliado a la evaluación de otros riesgos psicosociales, en este caso el burnout o síndrome de estar quemado, y la consideración de otros factores positivos implicados en la percepción de calidad de vida laboral, como el engagement o la vinculación personal con el trabajo, mostrando que los trabajadores con discapacidad están expuestos a riesgos psicosociales como los mencionados en igual medida que la población general, y que el modelo demandas- recursos en el trabajo resulta un marco conceptual adecuado para el análisis y la prevención de estos procesos.

La Agencia Europea para la Seguridad y la Salud en el Trabajo establece una serie de recomendaciones específicas para la prevención de riesgos en los trabajadores con discapacidad ${ }^{86}$. En primer lugar, recomienda tener en cuenta las diferencias individuales de los trabajadores, evitando las generalizaciones y las suposiciones acerca de los riesgos para la salud y la seguridad asociados a una discapacidad determinada. En concreto, sugiere lo siguiente:

- identificar grupos de trabajadores que podrían correr un mayor riesgo;

- realizar una evaluación específica de los riesgos que corren, teniendo en cuenta tanto la naturaleza y alcance de la discapacidad como el entorno de trabajo;

- tener en cuenta las capacidades específicas de las personas a la hora de planificar el trabajo; los trabajadores con discapacidad suelen tener habilidades especiales, que no deberían desperdiciarse debido a unas condiciones de trabajo mal o insuficientemente adaptadas;

- consultar a las personas implicadas durante el proceso de evaluación de riesgos, y consultarlas acerca de sus percepciones y experiencias concretas;

- solicitar asesoramiento cuando sea necesario; por ejemplo, a las autoridades y servicios de seguridad y salud en el trabajo, a los expertos en salud ocupacional y en ergonomía, y a los servicios de empleo para personas con discapacidad o las organizaciones de discapacitados.

Además, este organismo aconseja adoptar las medidas pertinentes dirigidas a la prevención de riesgos en al menos los siguientes aspectos: entorno de trabajo (accesibilidad, condiciones ambientales, soporte técnico), colocación de señales, comunicación (información adaptada sobre prevención de riesgos), organización del trabajo y de las tareas, horario de trabajo, formación y supervisión, promoción y traslado, y procedimientos de emergencia adaptados a todas los tipos de discapacidad. Adicionalmente, la Agencia recomienda la consulta a los trabajadores, una práctica aún más relevante en este caso que en el de los trabajadores sin discapacidad, así como la formación en materia de igualdad que permita la prevención y detección de posibles actitudes y conductas de discriminación en los lugares de trabajo, un aspecto especialmente importante en el caso de los riesgos de carácter psicosocial. 
Un aspecto que requiere atención específica es el relativo a la prevención de todo tipo de acoso en el trabajo -mobbing, sexual, etc.- dirigido hacia las personas con discapacidad, así como al establecimiento de mecanismos de detección y de sanción eficaces cuando se produzcan. Este colectivo puede ser más vulnerable aún que el de los empleados sin discapacidad a este tipo de comportamientos de abuso, de hostigamiento y de daño psicológico o moral, y es posible que carezca tanto de habilidades para detectarlo como de estrategias de afrontamiento eficaces, por lo que resulta ineludible para los responsables de la organización, los supervisores y los compañeros la adopción de medidas que prevengan este tipo de riesgo psicosocial. Por ejemplo, un reciente estudio realizado en el sector público en el Reino Unido ${ }^{87}$ ha mostrado cómo el propio proceso de ajuste y de adaptación al puesto de trabajo de las personas con discapacidad puede convertirse en una experiencia de acoso por parte del responsable o supervisor y derivar en estrés y en mala salud de los empleados a pesar de que les asistan derechos legales y los trabajadores estén obligados a adoptar las medidas de adaptación exigidas por la ley.

Por último, quisiéramos destacar un aspecto acerca del que no conocemos que se hayan realizado estudios específicos, como es el de los trabajadores de edad y la anticipación de algún tipo de posible discapacidad, salvo en sus aspectos preventivos para la salud general ${ }^{88}$ y la evaluación de su capacidad funcional ${ }^{89}$. En el caso específico al que nos referimos, se trata de posibles riesgos para el bienestar y la salud de personas actualmente sin discapacidad, pero cuya edad les sitúa ante la posibilidad de desarrollar o adquirir una discapacidad en un futuro más o menos cercano, como puede ser reducción o pérdida de movilidad total o parcial, reducción de capacidades sensoriales o cognitivas, etc. Estos trabajadores pueden ser mucho más vulnerables a riesgos de carácter psicosocial (estrés, acoso, tensión y sobrecarga de trabajo) debido a su condición de posible o probable empleado con discapacidad, lo que puede presionarles a cambiar de trabajo, abandonar la organización o aceptar ofertas de prejubilación o retiro laboral anticipado en contra de su voluntad, o al menos bajo condiciones de pérdida de control y experiencias de indefensión antes situaciones laborales aversivas, como hemos comprobado en estudios realizados con trabajadores de edad y salida anticipada del mercado laboral ${ }^{90}$. En este sentido, los modelos teóricos y la literatura sobre reestructuraciones y reducciones de plantilla -downsizing- pueden contribuir al análisis de este tipo específico de riesgos psicosociales y de discriminación laboral que combinan en su objetivo la edad con la (anticipada) discapacidad, y que creemos que pueden tener a su vez importaciones repercusiones considerando las diferencias de género y las diferencias ocupacionales en distintos sectores de actividad.

\section{CONCLUSIONES}

El denominado mercado accesible de la década de 1990, que permitió y facilitó un significativo incremento en el acceso de las personas con discapacidad al mercado laboral integrado, ha sido objeto en los primeros años del siglo XXI de importantes desafíos, turbulencias e incertidumbres ${ }^{61}$, que se han agravado con la actual crisis presupuestaria, financiera y económica global.

Esta situación externa, pero que afecta a las políticas y prácticas organizacionales concretas en buena parte de los países, viene a sumarse a las barreras y dificultades que a pesar de los indudable avances logrados en las dos últimas décadas aún persisten y que dificultan o retrasan la plena normalización de las personas con discapacidad en el mercado laboral ${ }^{13}$. Aunque los datos disponibles no son completamente fiables, la realidad es que las tasas de personas con discapacidad empleadas continúan proporcionalmente muy por debajo de las de la población general ${ }^{21,19,15,91}$.

Los resultados del estudio de Bruyère y sus colaboradores ${ }^{11}$ muestran que las principales y más eficaces medidas para eliminar barreras en el acceso y en la integración laboral de las personas con discapacidad en contextos no segregados consistían en un 
mayor compromiso por parte de la alta dirección en el diseño y aplicación de políticas y práctica eficaces; la implantación de programas de formación y cualificación de los empleados; la creación de servicios de counseling y apoyo técnico a las personas con discapacidad, así como programas de mentoring; y el incremento de ayuda fiscales e incentivos a la contratación de personas con discapacidad. Sin embargo, como ha señalado Foster $^{87}$, lamentablemente en ocasiones la realidad a la que se enfrentan las personas con discapacidad en su proceso de integración laboral es el resultado de una lotería, es decir, que el supervisor o el responsable de la organización tenga a bien negociar individualmente con el empleado las medidas de adaptación y acomodación necesarias, en lugar de tomar las decisiones pertinentes a las que le obliga la ley.

Por su parte, en momentos de cambio como los actuales y con un mercado laboral frágil e inestable, la rápida expansión, la mejora en el acceso y el perfeccionamiento de las Tecnologías de la Información y la Comunicación, así como sus desarrollos en el futuro próximo, pueden contribuir también a la implementación de sistemas y programas de teletrabajo que permitan el acceso al mercado laboral de personas con ciertos tipos de discapacidad ${ }^{92}$ y en contextos rurales o con pocas oportunidades de empleo presencial, en una proporción mayor de la que se ha producido hasta ahora.

Otro aspecto que requiere consideración y una mayor atención en el futuro es el referido a la eventualidad de una discapacidad sobrevenida a lo largo de la carrera profesional, cuyo origen sea, o no, la propia actividad laboral, y las posibles barreras a la reincorporación de la persona con discapacidad a su puesto anterior o a otro en la misma organización ${ }^{93,94}$. Estos procesos resultan especialmente importantes si tenemos en cuenta el progresivo envejecimiento de la población trabajadora y el retraso en la edad de jubilación obligatoria que están propiciando los gobiernos de diversos países, entre ellos España, ya que en cierta medida todo ello puede incrementar en las próximas décadas el número de personas con discapacidad, congénita o sobrevenida, que acceden, se mantienen o se reincorporan al mercado laboral.

Por último, nos parece necesario recordar que aunque las políticas de igualdad, de no discriminación, de fomento del empleo y del ejercicio de la ciudadanía plena, así como la creciente sensibilidad de las organizaciones hacia la gestión de la diversidad favorecida por el movimiento de la Responsabilidad social corporativa ${ }^{71}$, se han traducido en indudables mejoras y avances sustanciales en el acceso, la integración, la normalización y la calidad de vida laboral de las personas con discapacidad, es cierto que aún queda mucho por hacer ${ }^{42}$, y en situaciones como la actual, donde muchos colectivos de trabajadores asisten a un retroceso en los derechos laborales y en las condiciones de trabajo, resulta indispensable mantener la vigilancia y continuar avanzando en este camino, algo de lo que todos, trabajadores con y sin discapacidad, somos responsables.

\section{REFERENCIAS BIBLIOGRÁFICAS}

1. Stone DL, Colella A. A model of factors affecting the treatment of disabled individuals in organization. AMR 1996; 21: 352-401.

2. McMahon B, Shaw L. Workplace discrimination and disability. J Vocat Rehabilitation 2005; 23: 137-145.

3. Nkomo SM. Discrimination. En J Barling y CL Cooper Eds, The SAGE Handbook of Organizacional Behavior. Volume 1: Micro Approaches, pp. 657-672. Londres: Sage; 2008.

4. Shore LM, Cheng-Herrera BG, Dean MA., Ehrhart KH, Jung DI, Randel AE, Singh G. Diversity in organizations: Where are we now and where are we going? Hum Resour Manag Rev 2009; 19: 117-133.

5. Franche RL, Baril R, Shaw W, Nicholas M, Loisel P. Workplace-based return-to-work interventions: optimizing the role of stakeholders in implementation and research. J Occupat Rehabilitation 2005; 15: 525-542.

6. Nafukho FM, Roessler RT, Kacirek K. Disability as a diversity factor: Implications for human resource practices. ADHR 2010; 12: 395-406.

7. Colella A, DeNisi AS, Varma A. Appraising the performance of employees with disabilities: a review and model. Hum Resour Manag Rev 1997; 7: 27-53. 
8. Colella A, Stone DL. Workplace discrimination toward persons with disabilities: a call for some new research directions. En R. Dipboye y A. Colella Eds., Discrimination at Work: The Psychological and Organizational Bases, pp. 227-253. Mahwah, NJ: Lawrence Erlbaum; 2005.

9. Winance M. Being normally different? Changes to normalization processes: from alignment to work on the norm. Disability \& Society 2007; 22: 625-638.

10. Baldridge DC, Veiga JF. The impact of anticipated social consequences on recurring disability accommodation requests. J Manag, 2006; 32: 158-179.

11. Bruyère $S M$, Erickson WA, VanLooy $S$. Comparative study of workplace policy and practices contributing to disability non-discrimination. Rehabilitation Psych 2004; 49: 28-38.

12. Irvine A, Lupart J. Into the workforce: Employers' perspectives of inclusion. Developmental Disab Bull 2008; 36: 225-250.

13. Ren LR, Paetzold RL, Collella A. A meta-analysis of experimental studies on the effects of disability on human resources judgments. Hum Resour Manag Rev 2008; 18: 191-203.

14. Stone-Romero EF, Stone DL. How do organizational justice concepts relate to discrimination and prejudice? En J. Greenberg y J. A. Colquitt Eds., Handbook of Organizational Justice, pp. 439-467. Mahwah, NJ: Lawrence Erlbaum; 2005.

15. Stone-Romero EF, Stone DL, Lukaszewski K. The influence of disability on role-taking in organizations. En A. M. Konrad, P. Prasad y J. K. Pringle Eds., Handbook of Workplace Diversity, pp. 401-425. Londres: Sage; 2006

16. Larson JE, Ryan CB, Wassel AK, Kaszynski KL, Ibara L, Glenn TL, Boyle MG. Analyses of employment incentives and barriers for individuals with psychiatric disabilities. Rehabilitation Psych 2011; 56: 145-149.

17. Kennedy RB, Harris NK. Employing persons with severe disabilities: Much work remains to be done. J Employment Couns 2005; 42: 133-139.

18. Crowther RE, Marshall M, Bond GR, Huxley P. Helping people with severe mental illness to obtain work: A systematic review. BMJ 2001; 322: 204-208.

19. Kaye HS. Stuck at the bottom rung: Occupational characteristics of workers with disabilities. J Occupat Rehabilitation 2009; 19: 115-128.

20. CERMI. Derechos Humanos y Discapacidad. Informe España 2010. Madrid: CERMI. Disponible en URL: http://www.cermi.es/es-ES/Biblioteca/Lists/Publicaciones/Attachments/267/Derecho\%20Humanos\%20 y\%20Discapacidad $\% 202010 \% 20 \mathrm{n} \% \mathrm{c} 2 \% \mathrm{ba} \% 205$.pdf

21. OED. Informe Olivenza 2010. Las personas con discapacidad en España. Olivenza, Badajoz: Observatorio Estatal de la Discapacidad. Disponible en URL: http://www.observatoriodeladiscapacidad.es/ informacion/documento/20110107/informe-olivenza-2010

22. Bell BS, Klein K. Effects of disability, gender, and job level on ratings of job applicants. Rehabilitation Psych 2001; 46: 229-246. Disponible en URL http://digitalcommons.ilr.cornell.edu/hrpubs/10

23. Graffam J, Shinkfield A, Smith K, Polzina U. Factors that influence employer decisions in hiring and retaining an employee with a disability. J Vocat Rehabilitation 2002; 17: 175-181.

24. Smith K, Webbe L, Graffam J., Wilson C. Employer satisfaction, job-match and future hiring intentions for employees with a disability, J Vocat Rehabilitation 2004; 21 (3): 165-173.

25. CERMI. Un Plan de Empleo de las personas con discapacidad en el siglo XXI. Madrid: CERMI; 2001.

26. Alcover CM, Martínez D, Zambrano Z. Efectos de las oportunidades de incorporación al mercado laboral sobre el desarrollo del contrato psicológico y las actitudes hacia el trabajo: el caso de los trabajadores con discapacidad en el sector de call/contact centres. Psicologia, Revista da Associação Portuguesa de Psicologia 2007; 21: 151-176.

27. Lahera J. Acceso al mercado laboral y contratación de los discapacitados. En, F. Valdés dir. y J. Lahera coord. , Relaciones Laborales de las personas con discapacidad. pp. 89-140. Madrid: Biblioteca Nueva, Fundación Largo Caballero, Fundación ONCE y Fundación Ortega y Gasset; 2005.

28. Colella A, Paetzold RL, Belliveau MA. Factors affecting coworkers' procedural justice inferences of the workplace accommodations of employees with disabilities. Personnel Psychology 2004; 57: 1-23.

29. Landy FJ, Conte JM. Work in the 21st Century. An Introduction to Industrial and Organizational Psychology. Boston, MA: McGraw-Hill; 2004.

30. Spataro S. Diversity in context: how organizational culture shapes reactions to workers with disabilities and others who are demographically different. Beh Sci \& Law 2005; 23 (special issue): 21-38. doi: $10.1002 /$ bsl.623

31. Gilbride D, Stensrud R, Vandergoot D, Golden K. Identification of the characteristics of work environments and employers open to hiring and accommodating people with disabilities. Rehabilitation Couns Bull 2003; 46 (3): 130-137. 
32. Schur L, Kruse D, Blanck P. Corporate culture and the employment of persons with disabilities. Beh Sci \& Law 2005; 23 (special issue): 3-20. doi: 10.1002/bsl.624

33. Triana MC, García MF, Colella A. Managing diversity: how organizational efforts to support diversity moderate the effects of perceived racial discrimination on affective commitment. Personnel Psychology 2010; 63 (4): 817-843.

34. Cubero CG. Situational leadership and persons with disabilities. Work 2007; 29: 351-356

35. Cesare SJ, Tannenbaum RJ, Dalessio A. Interviewers' decisions related to applicant handicap type and rater empathy. Human Performance 1990; 3: 157-171.

36. Jones BJ, Gallagher BJ, Kelley JM, Massari L0. A survey of Fortune 500 corporate policies concerning the psychiatrically handicapped. J Rehabilitation 1991; 57: 31-35.

37. Macan TH, Hayes TL. Both sides of the employment interview interaction: Perceptions of interviewers and applicants with disabilities. Rehabilitation Psych 1995; 40: 261-278.

38. Schweitzer NJ, Deely J. Interviewing the disabled job applicant. Personnel Journal 1982; 61: 205-209.

39. Fundación ONCE y Fundación MANPOWER. Perspectivas del mundo empresarial, respecto a la contratación de personas con discapacidad. [Informe]. Madrid: Autor; 2008.

40. Scherbaum Ch. A, Scherbaum KL, Popovich P. Predicting job-related expectancies and affective reactions to employees with disabilities from previous work experience. J App Soc Psych 2005; 35 (5): 889-904.

41. McFarlin DB, Song J, Sonntag M. Integrating the disabled into the work force: A survey of Fortune 500 company attitudes and practices. Employee Responsibilities \& Rights J 1991; 4: 197-123.

42. Sayce L. Beyond goods intentions. Making anti-discrimination strategies work. Disability \& Society 2003; 18: 625-642.

43. Link BG, Phelan JC. On the nature and consequences of stigma. Annual Review of Sociology 2001; 27: 363-385.

44. McLaughlin ME, Bell MP, Stringer DY. Stigma and acceptance of persons with disabilities. Understudied aspects of workforce diversity. Gr \& Org Manag 2004; 29: 302-333.

45. Berry JO, Meyer JA. Employing people with disabilities: Impact of attitude and situation. Rehabilitation Psych 1995; 40: 211-222

46. Crudden A, McBroom LW. Barriers to employment: A survey of employed persons who are visually impaired. J Visual Impairment \& Blindness 1999; 93: 341-350.

47. Havranek JE. The social and individual costs of negative attitudes toward persons with physical disabilities. J App Rehabilitation Couns 1991; 22:15-21.

48. Lawthom R. Against all odds: Managing diversity. En N. Chmiel ed., Introduction to Work and Organizational Psychology: A European Perspective, pp. 387-406. Oxford: Blackwell; 2000.

49. Thomas A. The multidimensional character of biased perceptions of individuals with disabilities. J Rehabilitation 2001; 67: 3-9.

50. Jones G E, Stone DL. Perceived discomfort associated with working with persons with varying disabilities. Perc \& Motor Skills 1995; 81: 911-919.

51. Livneh $\mathrm{H}$. On the origin of negative attitudes toward people with disabilities. Rehabilitation Literature 1982; 43: 338-347.

52. Popovich P, Scherbaum Ch. A, Scherbaum K L, Polinko N. The assessment of attitudes towards individuals with disabilities in the workplace. J of Psychology 2003; 137: 163-177.

53. LaRocco JM, House JS, French JRP. Social support, occupational stress, and health. J Health Social Behav 1980; 21: 202-218.

54. Eisenberger R, Huntington R, Huntington S, Sowa D. Perceived organizacional support. J App Psych 1986; 71: 500-507.

55. Beehr TA, Jex SM, Stacy BA, Murray MA. Work stressors and coworker support as predictors of individual strain and job performance. J Organ Behav 2000; 21: 391-405.

56. Storey K. A review or research on natural support interventions in the workplace for people with disabilities. International J Rehabilitation Research 2003; 26: 79-84.

57. Chadsey JG. Promoting social relationships in employment settings through natural support strategies. Giornale Italiano delle Disabilita 2004; 4: 11-21.

58. Cimera RE. Utilizing co-workers as natural supports. J Disability Policy Studies 2001; 11: 194-201.

59. Murphy ST, Rogan PM. Developing Natural Supports in the Workplace: A Practitioner's Guide. St Augustine: Training Resource Network; 1994. 
60. Storey K, Certo N J. Natural supports for increasing integration in the workplace for people with disabilities: a review of the literature and guidelines for implementation. Rehabilitation Couns Bull 1996; 40: 62-76.

61. Bricourt JC. Partnering with the 21st century workplace: Leveraging workplace ecology. Work: J Prevention, Assessment \& Rehabilitation 2003; 21: 45-56.

62. Butterworth J, Hagner D, Kielnan W E, Schalock R L. Natural supports in the workplace and community: defining an agenda for research and practice. J Association Persons Severe Handicaps 1996; 21:103-113.

63. Jenaro C, Mank D, Bottomley J, Doose S, Tuckerman P. Supported employment in the international context: An analysis of processes and outcomes. J Vocat Rehabilitation 2002; 17: 5-21.

64. McHugh SH, Storey K, Certo NJ. Training job coaches to use natural support strategies. J Vocat Rehabilitation 2002; 17: 155-163.

65. Chadsey J, Beyer S. Social Relations in the Workplace. Mental Retar \& Develop Disabilities 2001; 7: 128-133.

66. Beehr TA, Drexler JA. Social support, autonomy, and hierarchical level as moderators of the role characteristics-outcome relationship. J Occupat Behav 1986; 7: 207-214.

67. Chiaburu D, Harrison D. Do peers make the place? Synthesis and metaanalysis of co-worker effects on perceptions, attitudes, OCBs and performance. J App Psych 2008; 93: 1082-1103.

68. Ng TW, Sorensen KL. Toward a further understanding of the relationships between perceptions of support and work attitudes: A meta-analysis. Gr \& Org Manag 2008; 33: 243-268.

69. Verdugo MA, Jordán de Urríes FB. Investigación sobre características del empleo con apoyo y resultados en diferentes variables. En M. A. Verdugo y F. B. Jordán de Urríes, coords., Hacia la integración plena mediante el empleo. Actas del VI Simposio Internacional de Empleo con Apoyo. Publicaciones del INICO; 2002.

70. Flores N, Jenaro C, Orgaz MB, Martín MV. Understanding quality of working life of workers with intellectual disabilities. JARID 2011; 24: 133-141.

71. Pérez V, Alcover $\mathrm{CM}^{\mathrm{a}}$. Apoyo social, satisfacción laboral y abandono en trabajadores con discapacidad. Boletín de Psicología 2011; 102: 23-41.

72. Carlson DS, Kacmar KM, Wayne JH, Grzywacz JG. Measuring the positive side of the work-family interface: Development and validation of a work-family enrichment scale. J Vocational Behav 2006; 68: 131-164.

73. Greenhaus J H, Powell GN. When work and family are allies: A theory of work and family enrichment. Acad of Manag J 2006; 31: 72-92.

74. Viswesvaran C, Sanchez JI, Fisher J. The role of social support in the process of work stress: A metaanalysis. J Vocational Behav 1999; 54: 314-334.

75. Jenaro C, Flores N, Arias B. Burnout and Coping in Human Service Practitioners. Prof Psych: Res \& and Prac 2007; 38: 80-87.

76. Kowalski C, Driller E, Ernstmann N, Alich S, Karbach U, Ommen O, et al. Associations between emotional exhaustion, social capital, workload, and latitude in decision-making among professionals working with people with disabilities. Research in Developmental Disab 2009; 31: 470-479.

77. Mutkins E, Brown, RF, Thornsteinsson E B. Stress, depression, workplace and social supports and burnout in intellectual disability support staff. J Intellect Disab Research 2011; 55: 500-510.

78. Lunsky Y, Bramston P. A preliminary study of perceived stress in adults with intellectual disabilities according to self-report and informant ratings. J Intellect \& Developmental Disab 2006; 31: 20-27.

79. Merz MA, Bricout J, Kock L. Disabilities and job stress: implications for vocational Rehabilitation planning. Work: J Prevent Assessment Rehabilitation 2001; 17: 85-96.

80. Verdugo MA, Jordán de Urríes B, Jenaro C, Caballo MC, Crespo M. Quality of life of workers with an intellectual disability in supported employment. J App Research Intellect Disab 2006; 19: 309-316.

81. Jenaro C, Flores N. Evaluación de la Calidad de Vida Laboral de trabajadores con discapacidad intelectual en empleo protegido: salud y riesgos psicosociales. Madrid: Real Patronato sobre Discapacidad. Ministerio de Trabajo y Asuntos Sociales; 2005.

82. Bakker AB, Demerouti E. The Job Demands-Resources model: State of the art. J Manag Psych 2008; 22: 309-328.

83. Demerouti E, Bakker AB, Nachreiner F, Schaufeli W B. The Job Demands-Resources model of burnout. J App Psych 2001; 86: 499-512.

84. Jenaro C, Flores N, Caballo C, Arias B, De Elena J. Demandas y riesgos psicosociales en trabajadores con discapacidad intelectual. Comunicación e Cidadanía 2006; 2: 1-21. 
85. Flores N, Jenaro C, González-Gil F, García-Calvo PM. Análisis de la calidad de vida laboral en trabajadores con discapacidad. Zerbitzuan: Gizarte serbitzuetarako aldizkaria, Revista de Servicios Sociales 2010; 47: 95-107.

86. Agencia Europea para la Seguridad y la Salud en el Trabajo. Garantizar la seguridad y la salud de los trabajadores con discapacidad. Factsheet, $\mathrm{n}^{\circ}$ 53. Disponible en URL: http://osha.europa.eu/es/ publications/factsheets/53

87. Foster D. Legal obligation or personal lottery? Employee experiences of disability and the negotiation of adjustments in the public sector workplace. Work, Employ \& Soc 2007; 21: 67-84.

88. Gimm G, Ireys H, Gilman B., Croake S. Impacto of early intervention programs for working adults with potentially disabling conditions: Evidence from the national DMIE. J Vocat Rehabilitation 2011; 34: 71-78.

89. León B. de, Díaz S. Revisión bibliográfica de la capacidad funcional en trabajadores de más de 65 años. Medicina y Seguridad del Trabajo 2011; 57: 63-76.

90. Alcover CM, Crego A. Factores implicados en la decisión de retiro laboral temprano: aproximaciones desde el análisis del discurso de una muestra de prejubilados españoles. Rev de Psic Soc Aplic 2005; 15: 133-163

91. Taylor H. Conflicting trends in employment of people with disabilities 1986-2000. The Harris Poll 2000; 59.

92. Bromet ES, Growick B. Telework: A new frontier in vocational Rehabilitation. J App Rehabilitation Couns 2001; 32: 19-24.

93. Blakwell TL, Leierer SJ, Haup, S, Kampitsis A. Predictors of vocational Rehabilitation return-to-work outcomes in workers' compensation. Rehabilitation Couns Bull 2001; 46: 108-114.

94. Martz E, Schiro-Geist C, Broadbent ED, Crandall LA. Predicting employment outcomes of individuals with disabilities: Attitudes and perceived barriers to work. J Appl Rehabilitation Couns 2010; 41: 36-41.

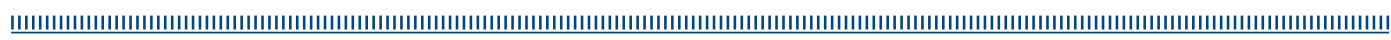

